Herz $2017 \cdot 42: 713-714$

https://doi.org/10.1007/s00059-017-4644-4

Online publiziert: 3. November 2017

๑) Springer Medizin Verlag GmbH 2017

CrossMark

\author{
R. Dörr' $\cdot$ B. Maisch ${ }^{2}$ \\ 'Praxisklinik Herz und Gefäße, Dresden, Deutschland \\ ${ }^{2}$ Herz- und Gefäßzentrum Marburg, Marburg, Deutschland
}

\title{
ESC-Leitlinien 2017
}

\section{Was ist neu und was ist besonders wichtig?}

Liebe Leser,

Ende August 2017 wurde beim Jahreskongress der European Society of Cardiology (ESC) in Barcelona eine Studie mit dem für dieses Leitlinienheft 8/2017 „wegweisenden“ Namen COMPASS vorgestellt [1]. Der Kompass (von italienisch „compasso“: Zirkel, Magnetnadel) ist das allseits bekannte Instrument zur Bestimmung einer fest vorgegebenen Himmelsrichtung oder eines Navigationskurses in der Seefahrt. Die Erkenntnis, dass sich Splitter von Magneteisenstein in die Nord-SüdRichtung drehen, war in Europa seit der griechischen Antike und in China seit der Zeit der Streitenden Reiche, zwischen 475 v. Chr. und 221 v. Chr. bekannt. In Europa beschrieb der englische Gelehrte Alexander Neckam 1187 den Kompass als eine magnetisierte schwimmende Nadel, die unter Seeleuten in Gebrauch war [2].

Eine ähnliche Kompassfunktion zur Orientierung bei schwierigen diagnostischen oder therapeutischen Entscheidungen in Praxis und Klinik haben die Leitlinien der internationalen kardiologischen Fachgesellschaften, des American College of Cardiology (ACC), der American Heart Association (AHA) und für Europa der ESC übernommen. Von der ESC wurden im August 2017 wiederum vier neue ESC Practice Guidelines zu den Themenbereichen „Management of Valvular Heart Disease“ (53 Seiten im Volltext, 56 Seiten als Pocket-Guidelines; [3]), „Diagnosis and Treatment of Peripheral Arterial Diseases“ (60 Seiten im Fulltext, 47 Seiten als Pocket Guidelines; [4]), „Management of Acute Myocardial Infarction in Patients Presenting with ST-
Segment Elevation“ (66 Seiten im Volltext, 56 Seiten als Pocket-Guidelines; [5]) und „2017 Focused Update on Dual Antiplatelet Therapy (DAPT)“ (48 Seiten im Volltext, 31 Seiten als Pocket-Guidelines; [6]) publiziert.

Allein der Textumfang dieser Leitlinien, deren Pocket-Versionen beim Leser ebenfalls eine gewisse Bibliophilie voraussetzen, erschwert die schnelle Erkennung von entscheidenden Neuerungen und essenziell wichtigen Komponenten.

Die Herausgeber dieses Leitlinienhefts 8/2017, das jeweils zum Jahresende erscheint und mittlerweile bereits eine langjährige Tradition besitzt, haben sich erneut das Ziel gesetzt, erfahrene deutschsprachige Koautoren dieser ESC Practice Guidelines bzw. deutschsprachige Experten für die entsprechenden Fachgebiete zu gewinnen. Die verkürzte deutschsprachige Interpretation soll helfen, diese Leitlinien leichter verständlich und im deutschen Sprachraum schneller bekannt $\mathrm{zu}$ machen. Entscheidend für den Leser sollte wiederum die Beantwortung der zentralen Fragen (Was ist neu? Was ist und bleibt besonders wichtig?) sein.

Haude kommentiert mit diesen Fragestellungen die ESC Guidelines „Management of Valvular Heart Disease " [3]. Im Bereich der kathetergestützten Therapieoptionen (TAVI) bei Aortenklappenstenose haben die in den letzten Jahren publizierten Ergebnisse von randomisierten Studien zu einer Neubewertung dieser Technologie geführt, die zu einer optionalen Indikationserweiterung auf $\mathrm{Pa}$ tienten mit nicht niedrigem konventionellen Operationsrisiko geführt hat. Im Bereich der Mitral- und Trikuspidalklappenerkrankungen sind die Empfehlun- gen für kathetergestützte Verfahren wegen der deutlich geringeren und teilweise nicht eindeutigen Datenlage zurückhaltender. Ein weiteres Augenmerk wurde auch auf die antithrombotische Begleittherapie gerichtet und die Etablierung von „Centers of Excellence“ für die Therapie der Herzklappenerkrankungen.

Espinola-Klein hat die ESC-Leitlinien „Diagnosis and Treatment of Peripheral Arterial Diseases“ [4] interpretiert. Wegen der hohen Koinzidenz der Atherosklerose sollte demnach bei atherosklerotischen Manifestationen in einer Gefäßprovinz gezielt nach weiteren Läsionen in anderen Gefäßregionen gesucht werden. Die Autorin betont, dass die Zahl randomisierter Studien bei der peripheren Atherosklerose geringer als bei der koronaren Herzkrankheit ist; dies gelte v. a. für die periphere arterielle Verschlusskrankheit (pAVK). Während die Empfehlungen zur Diagnostik und Klassifikation weitgehend etabliert sind, beruhen zahlreiche Empfehlungen der ESCLeitlinien „Peripheral Arterial Diseases“ nur auf Expertenmeinungen und Konsensusempfehlungen (Evidenzgrad C).

Von Thiele et al. wurden die ESC-Leitlinien „Management of Acute Myocardial Infarction in Patients Presenting with STSegment Elevation“ [5] bearbeitet. Die primäre perkutane Koronarintervention (PCI) bleibt die bevorzugte Reperfusionstherapie. Veränderungen hinsichtlich der Revaskularisation betreffen v. a. Empfehlungen zur Wahl des radialen $\mathrm{Zu}$ gangsweges, zur Verwendung von Drugeluting-Stents (DES) anstelle von Baremetal-Stents (BMS) sowie zur kompletten Revaskularisation und zur Vermeidung einer Thrombusaspiration. Bei der Begleittherapie während der PCI wurde 
Bivalirudin herabgestuft. Die Indikation für eine $\mathrm{O}_{2}$-Therapie ist nur noch gegeben bei einer $\mathrm{O}_{2}$-Sättigung von weniger als $90 \%$. Die intraaortale Ballonpumpe sollte im kardiogenen Schock nicht mehr verwendet werden. Neue Empfehlungen gibt es auch in Bezug auf die Dauer der dualen Antiplättchentherapie für Patienten, bei denen nach den initialen $12 \mathrm{Mo-}$ naten kein Blutungsereignis aufgetreten ist.

Schäfer et al. haben schließlich die Leitlinie „2017 Focused Update on Dual Antiplatelet Therapy (DAPT)“ [6] auf die wesentlichen Kernaussagen komprimiert. Auch bei hohem Blutungsrisiko wird der DES dem BMS vorgezogen. Die normale DAPT-Dauer nach elektiver PCI liegt bei 6 Monaten, nach akutem Koronarsyndrom (ACS) bei 12 Monaten. In begründeten Einzelfällen mit sehr hohem Blutungsrisiko kann auch nach Verwendung moderner DES eine DAPT bis auf 1 Monat reduziert werden. Die Dauer der DAPT wird dynamisch bewertet, was bedeutet, dass bei zuvor nicht absehbaren Blutungsereignissen unter DAPT deren finale Dauer neu angepasst werden muss. Zum Abschätzen des Blutungsrisikos wird per se das Verwenden von Scores wie dem PRECISEDAPT-Score empfohlen. Eine DAPT bei Patienten mit Vorhofflimmern und Indikation für eine orale Antikoagulation stellt weiterhin eine therapeutische Herausforderung dar, bei der eine Triple-Therapie grundsätzlich immer mit einem besonders hohen Blutungsrisiko verbunden ist.

Wir bedanken uns bei den Autoren der Zeitschrift Herz und wünschen allen Lesern erneut viel Vergnügen bei der Lektüre dieses Leitlinienhefts 8/2017. Trotz einer zunehmenden politischen Orientierungslosigkeit in dieser Welt, der der politische Kompass abhandengekommen $\mathrm{zu}$ sein scheint, wünschen wir allen Lesern ein gesegnetes Weihnachtsfest und ein hoffentlich friedvolles und gesundes neues Jahr 2018.

Ihre

Dr. med. Rolf Dörr

Prof. Dr. med. Bernhard Maisch

\section{Korrespondenzadresse}

\section{Dr. med. R. Dörr}

Praxisklinik Herz und Gefäße

Heinrich-Cotta-Str. 12, 01324 Dresden,

Deutschland

doerr@praxisklinik-dresden.de

Interessenkonflikt. R. Dörr und B. Maisch geben an, dass kein Interessenkonflikt besteht.

\section{Literatur}

1. Eikelboom JW, Connolly SJ, Bosch J et al (2017) 2017 COMPASS investigators. Rivaroxaban with or without aspirin in stable cardiovascular disease. N Engl J Med 377(14):1319-1330. https://doi.org/ 10.1056/NEJMoa1709118

2. Wolfschmidt G (2009) Sterne weisen den Weg-Geschichte der Navigation. Nuncius Hamburgensis Beiträge zur Geschichte der Naturwissenschaften, Bd. 15. Books on Demand, Norderstedt. ISBN 9783837039696

3. Baumgartner H, FalkV, Bax JJ et al (2017) 2017ESC/ EACTS guidelines for the management of valvular heart disease: the task force for the management of valvular heart disease of the European society of cardiology (ESC) and the European association for Cardio-thoracic surgery (EACTS). Eur Heart J. https://doi.org/10.1093/eurheartj/ehx391

4. Aboyans V, Ricco JB, Bartelink MEL et al (2017) 2017 ESC guidelines on the diagnosis and treatment of peripheral arterial diseases, in collaboration with the European society for vascular surgery (ESVS): document covering atherosclerotic disease of extracranial carotid and vertebral, mesenteric, renal, upper and lower extremity arteries endorsed by: the European stroke organization (ESO)the task force for the diagnosis and treatment of peripheral arterial diseases of the European society of cardiology (ESC) and of the European society for vascular surgery (ESVS). Eur Heart J. https://doi. org/10.1093/eurheartj/ehx095

5. Ibanez B, James S, Agewall S et al (2017) 2017 ESC Guidelines for the management of acute myocardial infarction in patients presenting with ST-segment elevation: The Task Force for the management of acute myocardial infarction in patients presenting with ST-segment elevation of the European Society of Cardiology (ESC). Eur Heart J.https://doi.org/10.1093/eurheartj/ehx393

6. Valgimigli M, Bueno H, Byrne RA et al (2017) 2017 ESC focused update on dual antiplatelet therapy in coronaryartery disease developed in collaboration with EACTS: The Task Force for dual antiplatelet therapy in coronary artery disease of the European Society of Cardiology (ESC) and of the European Association for Cardio-Thoracic Surgery (EACTS). Eur Heart J. https://doi.org/10.1093/eurheartj/ ehx419

\section{Autoren WERKSTATT}

GRATIS

Ein Service für Autoren von Springer Medizin

S

\section{Fortbildungen für Autoren und Gutachter}

Die ersten Veröffentlichungen sind für jeden Mediziner ein wichtiger Schritt in seiner Karriere als Wissenschaftler. Wissenschaftliche Artikel sind entscheidend dafür, dass die eigene Arbeit in der Community wahrgenommen wird. Es geht darum, die eigenen Ideen national und international auszutauschen und sicherzustellen, dass die Ergebnisse Wirkung erzielen. Die Online-Kurse der Autorenwerkstatt helfen, sich leicht einen Überblick über das Schreiben, Einreichen, Begutachten und Veröffentlichen eines Manuskripts zu verschaffen.

5 Online-Kurse zu den wichtigsten Standards des wissenschaftlichen Publizierens:

- Wie verfasse ich ein Manuskript?

- Writing in English für deutschsprachige Autoren

- Wie funktionieren Publikation und Begutachtung?

- Anleitung zur Open-AccessVeröffentlichung

- Leitfaden zur Peer-ReviewBegutachtung

Für alle, die auf SpringerMedizin.de registriert sind!

Jetzt gratis fortbilden unter www.springermedizin.de/ autorenwerkstatt/ 